\title{
Phase diagram of the two-dimensional ferromagnetic three-color Ashkin-Teller model
}

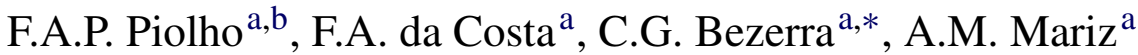 \\ ${ }^{a}$ Departamento de Física Teórica e Experimental, Universidade Federal do Rio Grande do Norte, 59072-970 Natal - RN, Brazil \\ ${ }^{\mathrm{b}}$ Departamento de Física, Universidade do Estado do Rio Grande do Norte, 59600-970 Mossoró - RN, Brazil
}

Received 17 August 2007; received in revised form 2 October 2007

Available online 13 November 2007

\begin{abstract}
In the present work we study the critical properties of the ferromagnetic three-color Ashkin-Teller model (3AT) by means of a Migdal-Kadanoff renormalization group approach on a diamond-like hierarchical lattice. The analysis of the fixed points and flux diagram of the recursion relations is used to determine the corresponding phase diagram (including its symmetry properties) and critical exponents. Our numerical results show the presence of four universality classes, three of them are associated to the Potts model with $q=2,4$ and 6 states. Finally, a connection between our findings and some known results from the literature is presented.
\end{abstract}

(c) 2007 Elsevier B.V. All rights reserved.

PACS: 05.10.Cc; 05.50.+q; 05.70.Fh; 64.60.-i

Keywords: Ising models; Ashkin-Teller; Renormalization group; Phase transitions; Phase diagrams

\section{Introduction}

The Ashkin-Teller (AT) model was introduced to study cooperative phenomena of quaternary alloys [1] on a lattice. Later, Fan has shown that the AT model could be described in a Hamiltonian form appropriated for localized spin magnetic systems [2] so that it can be considered as two superposed Ising models represented by the classical spin variables $\sigma$ and $\tau$, respectively. This version introduced by Fan is also known as two-color AT model due to the presence of the two Ising systems in the Hamiltonian. Since it was introduced, the AT model is one of the most studied systems in statistical mechanics due mainly to the richness of critical phenomena revealed by its phase diagrams both in two and three dimensions [3]. In the two-dimensional version, the model exhibits a line of fixed points along which the critical exponents vary continuously. This result was obtained through a mapping [4] onto the eight-vertex model solved by Baxter [5]. A number of exact results have been obtained for the isotropic AT model (IAT), which corresponds to the case where the two Ising systems are identical to each other, on a square lattice [6,7]. Many techniques have been employed in determining the corresponding phase diagram. For example,

\footnotetext{
* Corresponding author. Tel.: +55 843215 3793; fax: +55 8432153791.

E-mail address: cbezerra@dfte.ufrn.br (C.G. Bezerra).
} 
real-space renormalization-group analysis [8], mean-field renormalization-group approach and generalizations [911], and Monte Carlo simulations [12-14].

A different version of the AT model corresponds to the case where the two coupled Ising systems are non-identical, i.e., they present different exchange couplings. This version is called anisotropic AT model (AAT). Among the known results from the literature for the AAT model, we can list the work of Wu and Lin [15] that using exact duality relations determined the general topology of the phase diagram on a square lattice. Their results were supported later by the work of Domany and Riedel [16] that used a Migdal-Kadanoff renormalization group technique. In recent papers, Benyoussef and collaborators have investigated the AAT model using mean-field treatments and Monte Carlo simulations [17], and finite size scaling as well [18]. Also Bezerra et al. [19] have investigated the AAT model through a real-space renormalization-group approach applying the concept of transmissivity [20]. They presented a very complete phase diagram for the AAT model in which new phases, due to the symmetry breaking between the spins $\sigma$ and $\tau$, are present. More recently, the phase transitions of the AAT on a family of hierarchical lattices was studied by Le and Yang [21].

In general the N-color Ashkin-Teller model (NAT) is a generalization of the AT model consisting of N coupled Ising systems. Such kind of extension seems to be first considered by Grest and Widom [22] who considered a pairwise coupling by a four-spin interaction. However, the pairwise coupling between the Ising systems may include more than four-spin interaction as $N>2$. Grest and Widom [22] showed the existence of a first-order phase transition in two dimension for $N>2$ as long as the four-spin interaction coupling is ferromagnetic. By using a series of techniques such as first-order perturbation expansion, mean-field theory and Monte Carlo simulations, they obtained the phase diagram for the 3AT in two and three dimensions. The phase diagram of the two-dimensional NAT model was also considered by Martins and Drugowich de Felicio who proved that the model exhibits an Ising-like continuous transition line in the region where the system presents two phase transitions [23]. Goldschmidt [24] considered a twodimensional anisotropic $N=3$ AT version where the Ising systems are coupled by a four-spin interaction and obtained a Kosterlitz-Thouless multicritical point where the line of first-order phase transitions merge in a line of continuous phase transitions. The work of Goldschmidt was later supported by Monte Carlo simulations by Drugowich de Felicio and collaborators [25]. Considering also a 3AT model with bond impurities in two dimension Murthy [26] found that quenched randomness converts a first-order phase transition into a continuous one. The complex nature of the full NAT Hamiltonian makes it very difficult to analyze from either analytical or computational techniques. A simpler version of this generalization is the so-called three-color Ashkin-Teller model (3AT). Despite its simplicity, compared to the NAT model, the 3AT model may present a very rich critical behavior, as we will show later in this article. The aim of this work is to study the critical properties (phase diagram, critical points and exponents) of the 3AT model. The plan of this article is as follows. In Section 2, we present the model and the theoretical technique used to obtain the phase diagram which is based on a real-space renormalization-group approach. Section 3 is devoted to discuss the critical behavior of the model. Finally, the summary of our results are presented in Section 4.

\section{Physical model}

In this section we describe the Hamiltonian of the 3AT model on a lattice. The Hamiltonian of the A3AT model has the form

$$
\mathcal{H}=-\sum_{\langle i, j\rangle}\left[J_{1} \sigma_{i} \sigma_{j}+J_{2} \theta_{i} \theta_{j}+J_{3} \tau_{i} \tau_{j}+J_{4} \sigma_{i} \sigma_{j} \theta_{i} \theta_{j}+J_{5} \sigma_{i} \sigma_{j} \tau_{i} \tau_{j}+J_{6} \theta_{i} \theta_{j} \tau_{i} \tau_{j}+J_{7} \sigma_{i} \sigma_{j} \theta_{i} \theta_{j} \tau_{i} \tau_{j}\right]
$$

where $\sum_{\langle i, j\rangle}$ means that the sum is over nearest-neighbor sites $i$ and $j$ (which may be, if necessary, extended to more distant neighbors); $\sigma, \theta$ and $\tau$ are independent spin variables $(\sigma= \pm 1, \theta= \pm 1, \tau= \pm 1)$. Also $J_{1}, J_{2}$ and $J_{3}$ are the exchange couplings of each Ising system; $J_{4}, J_{5}$ and $J_{6}$ are the exchange couplings which couples pairs of independent Ising systems; and $J_{7}$ represents the six-spin interaction. The range of values of the exchange couplings considered here corresponds to the ferromagnetic model.

In order to address the 3 AT let us consider $J_{1}=J_{2}=J_{3}$ and $J_{4}=J_{5}=J_{6}$. The dimensionless Hamiltonian can be rewritten as

$$
\frac{\mathcal{H}}{k_{\mathrm{B}} T}=-\sum_{\langle i, j\rangle}\left[K_{2}\left(\sigma_{i} \sigma_{j}+\theta_{i} \theta_{j}+\tau_{i} \tau_{j}\right)+K_{4}\left(\sigma_{i} \sigma_{j} \theta_{i} \theta_{j}+\sigma_{i} \sigma_{j} \tau_{i} \tau_{j}+\theta_{i} \theta_{j} \tau_{i} \tau_{j}\right)+K_{6} \sigma_{i} \sigma_{j} \theta_{i} \theta_{j} \tau_{i} \tau_{j}\right]
$$




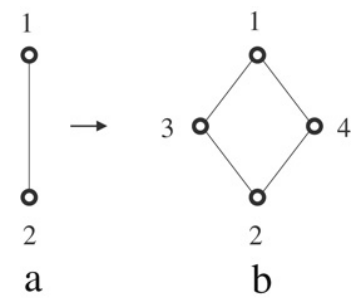

Fig. 1. The graph used for the generation of the hierarchical lattice considered in this work. The scaling factor $b=2$ and the number of bonds $m=4$ furnish a fractal dimension $d_{f}=2$.

Here $K_{2}=J_{1} / k_{\mathrm{B}} T=J_{2} / k_{\mathrm{B}} T=J_{3} / k_{\mathrm{B}} T$ is the dimensionless two-spin coupling, $K_{4}=J_{4} / k_{\mathrm{B}} T=J_{5} / k_{\mathrm{B}} T=$ $J_{6} / k_{\mathrm{B}} T$ is the dimensionless four-spin coupling, and $K_{6}=J_{7} / k_{\mathrm{B}} T$ is the dimensionless six-spin coupling. Also $k_{\mathrm{B}}$ and $T$ are the Boltzmann's constant and temperature, respectively.

The model described above will be studied in a hierarchical lattice generated by the graph shown in Fig. 1. For this purpose we will employ a real-space renormalization-group approach which is known to be exact for hierarchical lattices [27]. Besides, the results obtained from this method are known to be a good qualitative approximation for the phase diagram in more realistic Bravais lattices. To treat the Hamiltonian of Eq. (2) we apply the cluster renormalization group transformation indicated in Fig. 1. The renormalization group recursive relations are constructed, after the decimation of the spin variables associated to sites 3 and 4 , requiring that the partition function be preserved, i.e.,

$$
\exp \left(\mathcal{H}_{1,2} / k_{\mathrm{B}} T\right)=\exp \left(\mathcal{H}_{1,2,3,4} / k_{\mathrm{B}} T\right) .
$$

Here $\mathcal{H}_{1,2}$ and $\mathcal{H}_{1,2,3,4}$ are the Hamiltonians associated to Fig. 1(a), (b), respectively. Note that $\mathcal{H}_{1,2}$ includes an additive constant. From now on, we will use the transmissivity vector [20] which simplifies the calculation and it is more useful for presenting the phase diagram. The transmissivity vector is given by,

$$
\begin{aligned}
& t_{2}=\frac{1+\mathrm{e}^{\left(-2 K_{2}-4 K_{4}-2 K_{6}\right)}-\mathrm{e}^{\left(-4 K_{2}-4 K_{4}\right)}-\mathrm{e}^{\left(-6 K_{2}-2 K_{6}\right)}}{1+3 \mathrm{e}^{\left(-2 K_{2}-4 K_{4}-2 K_{6}\right)}+3 \mathrm{e}^{\left(-4 K_{2}-4 K_{4}\right)}+\mathrm{e}^{\left(-6 K_{2}-2 K_{6}\right)}}, \\
& t_{4}=\frac{1-\mathrm{e}^{\left(-2 K_{2}-4 K_{4}-2 K_{6}\right)}-\mathrm{e}^{\left(-4 K_{2}-4 K_{4}\right)}+\mathrm{e}^{\left(-6 K_{2}-2 K_{6}\right)}}{1+3 \mathrm{e}^{\left(-2 K_{2}-4 K_{4}-2 K_{6}\right)}+3 \mathrm{e}^{\left(-4 K_{2}-4 K_{4}\right)}+\mathrm{e}^{\left(-6 K_{2}-2 K_{6}\right)}}
\end{aligned}
$$

and

$$
t_{6}=\frac{1-3 \mathrm{e}^{\left(-2 K_{2}-4 K_{4}-2 K_{6}\right)}+3 \mathrm{e}^{\left(-4 K_{2}-4 K_{4}\right)}-\mathrm{e}^{\left(-6 K_{2}-2 K_{6}\right)}}{1+3 \mathrm{e}^{\left(-2 K_{2}-4 K_{4}-2 K_{6}\right)}+3 \mathrm{e}^{\left(-4 K_{2}-4 K_{4}\right)}+\mathrm{e}^{\left(-6 K_{2}-2 K_{6}\right)}} .
$$

We may also express the recursion relations, according to our convenience, in terms of the dual transmissivity vector defined by [19],

$$
\begin{aligned}
& t_{2}^{D}=\frac{1+t_{2}-t_{4}-t_{6}}{1+3 t_{2}+3 t_{4}+t_{6}}=\mathrm{e}^{\left(-2 K_{2}-4 K_{4}-2 K_{6}\right)}, \\
& t_{4}^{D}=\frac{1-t_{2}-t_{4}+t_{6}}{1+3 t_{2}+3 t_{4}+t_{6}}=\mathrm{e}^{\left(-4 K_{2}-4 K_{4}\right)},
\end{aligned}
$$

and

$$
t_{6}^{D}=\frac{1-3 t_{2}+3 t_{4}-t_{6}}{1+3 t_{2}+3 t_{4}+t_{6}}=\mathrm{e}^{\left(-6 K_{2}-2 K_{6}\right)} .
$$

Following the lines of Ref. [27], the following recursion relations are obtained

$$
t_{2}^{\prime D}=4\left[\frac{t_{2}^{D}+2 t_{2}^{D} t_{4}^{D}+t_{4}^{D} t_{6}^{D}}{1+3\left(t_{2}^{D}\right) 2+3\left(t_{4}^{D}\right) 2+\left(t_{6}^{D}\right) 2}\right]^{2},
$$




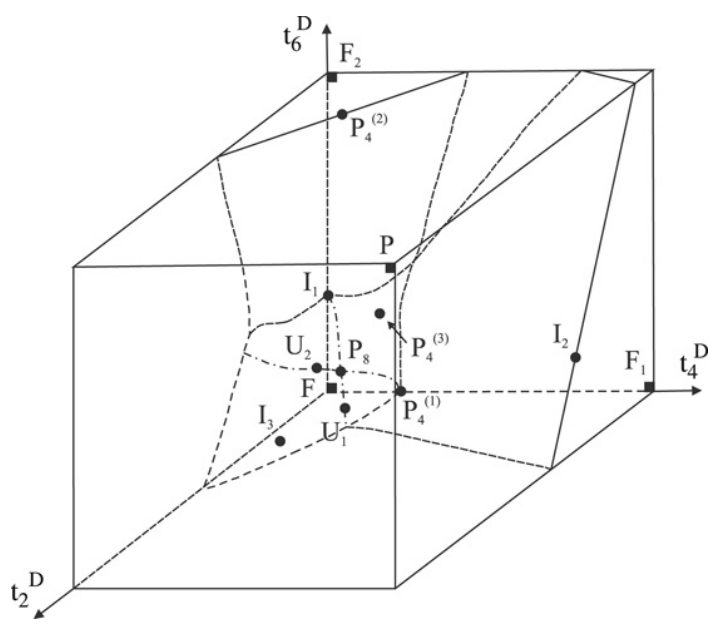

Fig. 2. Phase diagram of the 3 AT model in $\left(t_{2}^{D}, t_{4}^{D}, t_{6}^{D}\right)$ space.

Table 1

Unstable fixed points and their respective eigenvalues and critical exponents

\begin{tabular}{llll}
\hline Fixed point & $\left(t_{2}^{D}, t_{4}^{D}, t_{6}^{D}\right)$ & $\left(\lambda_{1}, \lambda_{2}, \lambda_{3}\right)$ & $v_{T}$ \\
\hline$P_{8}$ & $(0.15876,0.15876,0.15876)$ & $(2.04536,1.13968,1.13968)$ & 0.96866 \\
$P_{4}^{(1)}$ & $(0,0.22033,0)$ & $(1.85260,0,0)$ & 1.12416 \\
$P_{4}^{(2)}$ & $(0.22033,0.22033,1)$ & $(1.85260,0,0)$ & 1.12416 \\
$P_{4}^{(3)}$ & $(0.06513,0.22033,0.29560)$ & $(1.85260,1.67857,0.77743)$ & 1.12416 \\
$I_{1}$ & $(0,0,0.29560)$ & $(1.67857,0,0)$ & 1.33827 \\
$I_{2}$ & $(0.29560,1,0.29560)$ & $(1.67857,0,0)$ & 1.33827 \\
$I_{3}$ & $(0.29560,0.08738,0.02583)$ & $(1.67857,0.70440,0.29560)$ & 1.33827 \\
$U_{1}$ & $(0.17073,0.17073,0.09005)$ & $(2.01743,1.27130,0.85550)$ & 0.98764 \\
$U_{2}$ & $(0.18520,0.12534,0.18520)$ & $(2.01743,1.27130,0.85550)$ & 0.98764 \\
\hline
\end{tabular}

$$
t_{4}^{\prime D}=4\left[\frac{\left(t_{2}^{D}\right) 2+t_{2}^{D} t_{6}^{D}+t_{4}^{D}+\left(t_{4}^{D}\right) 2}{1+3\left(t_{2}^{D}\right) 2+3\left(t_{4}^{D}\right) 2+\left(t_{6}^{D}\right) 2}\right]^{2}
$$

and

$$
t_{6}^{\prime D}=4\left[\frac{t_{6}^{D}+3 t_{2}^{D} t_{4}^{D}}{1+3\left(t_{2}^{D}\right) 2+3\left(t_{4}^{D}\right) 2+\left(t_{6}^{D}\right) 2}\right]^{2} .
$$

These equations completely determine the phase diagram (see Fig. 2) and critical exponents (see Table 1) for the 3AT model. The structure of the hierarchical lattice based on the graph exhibited in Fig. 1 prevents us of studying antiferromagnetic configuration. Thus we will restrict our analysis to the ferromagnetic case which requires

$$
K_{2}+K_{4} \geq 0, \quad 3 K_{2}+K_{6} \geq 0, \quad K_{2}+2 K_{4}+K_{6} \geq 0,
$$

in order to obtain stable ferromagnetic phases in the present system, i.e., the ground state corresponds to ferromagnetic order.

\section{The phase diagram of the 3AT model}

From Eqs. (10)-(12) we numerically determined the existence of four magnetic phases separated by twodimensional critical surfaces. More specifically, these four magnetic phases are:

(i) paramagnetic (P) in which $\langle\sigma\rangle=\langle\theta\rangle=\langle\tau\rangle=0,\langle\sigma \theta\rangle=\langle\sigma \tau\rangle=\langle\theta \tau\rangle=0$ and $\langle\sigma \theta \tau\rangle=0$; 
Table 2

Location of the trivial fixed points and their respective magnetic phases

\begin{tabular}{lll}
\hline Trivial fixed point & $\left(t_{2}^{D}, t_{4}^{D}, t_{6}^{D}\right)$ & Magnetic phase \\
\hline$F$ & $(0,0,0)$ & Ferromagnetic \\
$F 1$ & $(0,1,0)$ & Intermediate one \\
$F 2$ & $(0,0,1)$ & Intermediate two \\
$P$ & $(1,1,1)$ & Paramagnetic \\
\hline
\end{tabular}

(ii) ferromagnetic (F) in which $\langle\sigma\rangle \neq 0,\langle\theta\rangle \neq 0,\langle\tau\rangle \neq 0,\langle\sigma \theta\rangle \neq 0,\langle\sigma \tau\rangle \neq 0,\langle\theta \tau\rangle \neq 0$ and $\langle\sigma \theta \tau\rangle \neq 0$;

(iii) intermediate one (F1) in which $\langle\sigma\rangle=\langle\theta\rangle=\langle\tau\rangle=0,\langle\sigma \theta\rangle=\langle\sigma \tau\rangle=\langle\theta \tau\rangle=0$ and $\langle\sigma \theta \tau\rangle \neq 0$; and

(iv) intermediate two (F2) in which $\langle\sigma\rangle=\langle\theta\rangle=\langle\tau\rangle=0,\langle\sigma \theta\rangle \neq 0,\langle\sigma \tau\rangle \neq 0,\langle\theta \tau\rangle \neq 0$ and $\langle\sigma \theta \tau\rangle=0$.

The trivial fixed points completely characterize the symmetries presented by the respective phases where those points are located, as shown in Fig. 2.

The corresponding phase diagram, in the three-dimensional space defined by the variables $t_{2}^{D}, t_{4}^{D}$ and $t_{6}^{D}$, is shown in Fig. 2. One can see in Fig. 2 that there are nine non-trivial fixed critical points, which are listed in Table 1 together with their critical exponents $v_{T}=\ln b / \ln \lambda_{1}$ and $\phi=\ln \lambda_{1} / \ln \lambda_{2}$. The non-trivial critical points are located on the two-dimensional critical surfaces. These surfaces are in fact boundaries of the attraction domains corresponding to the four trivial fixed points which characterize each magnetic phase. The trivial fixed points and the magnetic phases associated to them are listed in Table 2.

The fixed points $I_{1}, I_{2}$ and $I_{3}$ present only one relevant eigenvalue and they belong to the universality class of the Potts model with $q=2$ states, which means Ising. $I_{1}$ is located on the boundary surface between the ferromagnetic and the intermediate two phases, $I_{2}$ is located on the boundary surface between the paramagnetic and the intermediate one phases, and finally $I_{3}$ is located on the boundary surface between the ferromagnetic and the paramagnetic phases.

We can also find in Table 1 the fixed points labeled $P_{4}^{(1)}, P_{4}^{(2)}$ and $P_{4}^{(3)}$ which correspond to the Potts model with $q=4$ states. $P_{4}^{(1)}$ is located on the boundary surface between the ferromagnetic and the intermediate one phases, $P_{4}^{(2)}$ is located on the boundary surface between the paramagnetic and the intermediate two phases, and finally $P_{4}^{(3)}$ is located on the boundary surface between the ferromagnetic and the paramagnetic phases. One can see from Table 1 that $P_{4}^{(1)}$ and $P_{4}^{(2)}$ present one relevant eigenvalue, while $P_{4}^{(3)}$ presents two relevant eigenvalues.

The fixed point $P_{8}$ corresponds to the Potts model with $q=8$ states and it is located on the boundary surface between the ferromagnetic and the paramagnetic phases. It is completely unstable with three relevant eigenvalues. It is interesting to note that $P_{8}$ is in the intersection between the planes $t_{2}^{D}=t_{4}^{D}$ and $t_{2}^{D}=t_{6}^{D}$. Those planes are invariant under the renormalization group recursion relations (Eqs. (10)-(12)), i.e., a given point in the $\left(t_{2}^{D}, t_{4}^{D}, t_{6}^{D}\right)$ space that belongs to the plane $t_{2}^{D}=t_{4}^{D}$ (or $t_{2}^{D}=t_{6}^{D}$ ), after iterated in the renormalization process, furnish a new point which also belongs to the plane $t_{2}^{D}=t_{4}^{D}$ (or $t_{2}^{D}=t_{6}^{D}$ ). The planes $t_{2}^{D}=t_{4}^{D}$ and $t_{2}^{D}=t_{6}^{D}$ are better illustrated in Figs. 3 and 4 , respectively.

On the one hand, Fig. 3 shows the plane $t_{2}^{D}=t_{4}^{D}$, from which one can see the presence of three magnetic phases: (i) ferromagnetic, (ii) intermediate two and (iii) paramagnetic. We can also see three stable fixed points (characterizing each magnetic phase) and four unstable fixed points, namely $P_{4}^{(2)}, I_{1}, P_{8}$ and $U_{1}$. On the other hand, the plane $t_{2}^{D}=t_{6}^{D}$ is shown in Fig. 4. There are also three magnetic phases in this plane: (i) ferromagnetic, (ii) intermediate one and (iii) paramagnetic. As in Fig. 3, one can see three stable fixed points (characterizing each magnetic phase) and four unstable fixed points, namely $P_{4}^{(1)}, I_{2}, P_{8}$ and $U_{2}$. If we compare Figs. 3 and 4, it is very easy to see the presence of a strong symmetry between them. As a matter of the fact, there is a striking resemblance between the planes illustrated in Figs. 3 and 4 and the phase diagram of the isotropic Ashkin-Teller model (IAT) $[8,19]$.

From the resemblance between Figs. 3 and 4 and the phase diagram of the IAT model, we can discuss the unstable fixed points labeled $U_{1}$ and $U_{2}$ which are located on the boundary surface between the ferromagnetic and paramagnetic phases. It is known from the literature that in two dimensions the IAT model presents a line of fixed points whose critical exponents vary continuously [6]. This line, the so-called Baxter's line, is the boundary between the ferromagnetic and paramagnetic phases. Usually renormalization group approaches fail in reproducing Baxter's line. For example, in Refs. [8,19] only one fixed point, among infinite possibilities that compose the Baxter's line, is reproduced. From Table 1 we can see that $U_{1}$ and $U_{2}$ do not belong to any universality class of the Potts model 


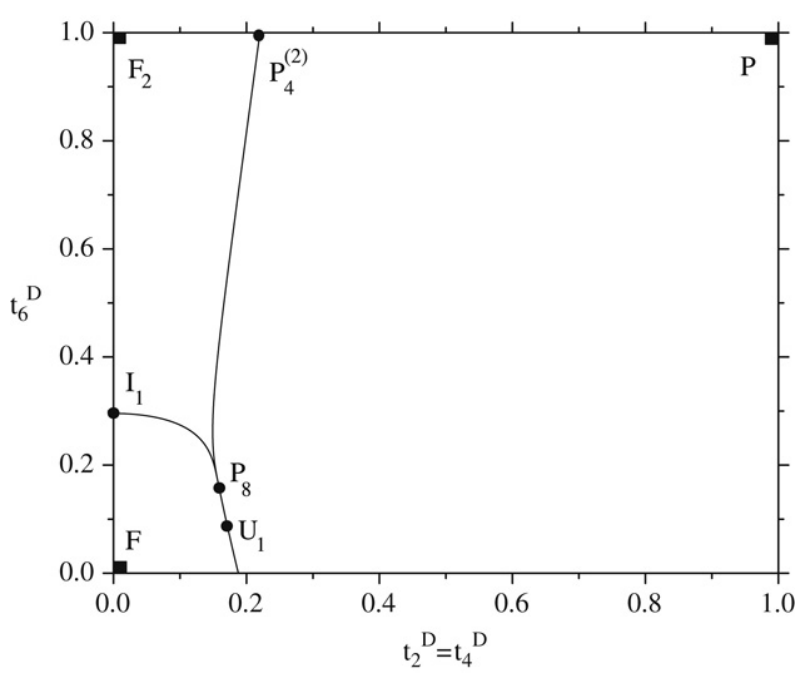

Fig. 3. Plane $t_{2}^{D}=t_{4}^{D}$ of Fig. 2 .

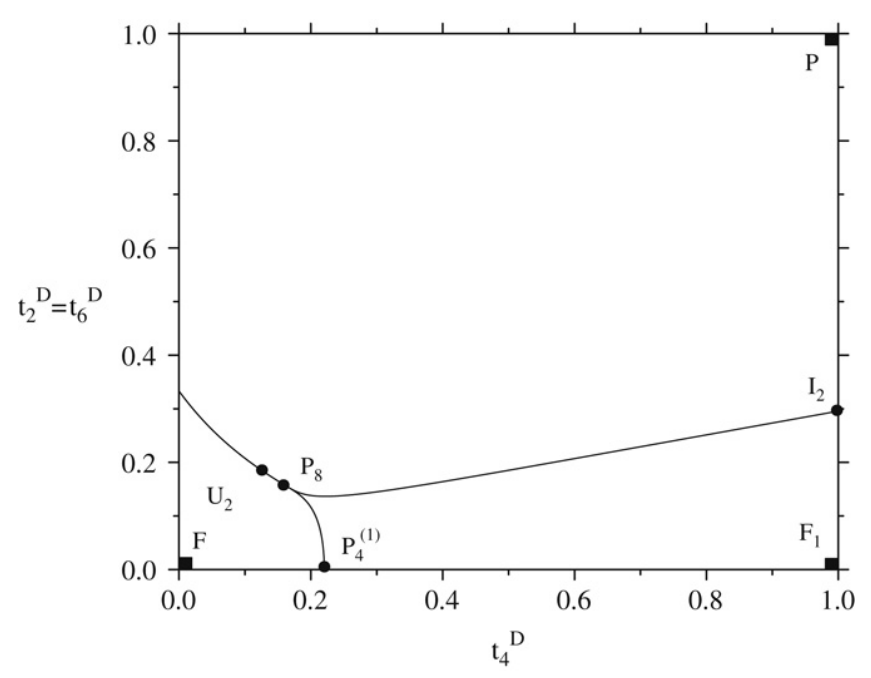

Fig. 4. Plane $t_{2}^{D}=t_{6}^{D}$ of Fig. 2 .

identified so far in this paper. We believe that the other methods as numerical simulations (work in progress), may be used to check if there is any trace of Baxter lines (along which the critical exponent vary continuously) associated with the points $U_{1}$ and $U_{2}$.

We believe that our results are a good qualitative representation of the phase diagram of the three-color Ashkin-Teller model on the square lattice. Unfortunately, like any renormalization group approach, the present one fails to detect some special features of the universality classes. In the following section we present our conclusions.

\section{Conclusions}

In summary, we have applied a Migdal-Kadanoff-like renormalization group scheme in order to determine the phase diagram and the critical behavior of the three-color Ashkin-Teller model on a diamond-type hierarchical lattice. Our results are exact for the hierarchical lattice generated by the graph shown in Fig. 1, but they may also be interpreted as a good qualitative representation for the 3AT model on the square lattice [28,29]. We have found the presence of four magnetic phases (listed in Table 2 along with their attractors) and nine unstable fixed points (listed in Table 1 along with their critical exponents). These nine unstable fixed points can be classified in four universality classes, 
three of them are associated to the Potts model with $q=2$ states $\left(I_{1}, I_{2}\right.$ and $\left.I_{3}\right), q=4$ states $\left(P_{4}^{(1)}, P_{4}^{(2)}\right.$ and $\left.P_{4}^{(3)}\right)$ and $q=8$ states $\left(P_{8}\right)$. Besides, there are two unstable fixed points $\left(U_{1}\right.$ and $\left.U_{2}\right)$ which do not belong to the universality class of the Potts model. We are presently investigating, through numerical simulations, if $U_{1}$ and $U_{2}$ are located on Baxter's lines, which our renormalization group approach fails in reproducing. Therefore, $U_{1}$ and $U_{2}$ could belong to one of the infinite universality classes which compose Baxter's line. We know that the results for the universality class can be improved if one considers more sophisticated graphs (for example, the wheatstone bridge) or systematically larger clusters $(b \rightarrow \infty)$. These tasks are currently in progress, and we intend to present them in a forthcoming publication.

\section{Acknowledgements}

We would like to thank Dr. J.M. de Araújo and Dr. I.S. Queiroz for fruitful discussions. We are in debt to Dr. A.O. Sousa for a critical reading of the manuscript. We also would like to thank the Brazilian Research Council CNPq for partial financial support.

\section{References}

[1] J. Ashkin, E. Teller, Phys. Rev. 64 (1943) 178.

[2] C. Fan, Phys. Lett. A 39 (1972) 136.

[3] R.V. Ditzian, J.R. Banavar, G.S. Grest, L.P. Kadanoff, Phys. Rev. B 22 (1980) 2542.

[4] L.P. Kadanoff, F.J. Wegner, Phys. Rev. B 4 (1971) 3989.

[5] R.J. Baxter, Phys. Rev. Lett. 26 (1971) 832.

[6] R.J. Baxter, Exactly Solved Models in Statistical Mechanics, Academic Press, London, 1982.

[7] B. Nienhuis, in: C. Domb, J.L. Lebowitz (Eds.), Phase Transitions and Critical Phenomena, vol. 11, Academic Press, London, 1987.

[8] A.M. Mariz, C. Tsallis, P. Fulco, Phys. Rev. B 32 (1985) 6055.

[9] J.A. Plascak, F.C. Sá Barreto, J. Phys. A 19 (1986) 2195.

[10] P.M.C. de Oliveira, F.C. Sá Barreto, J. Stat. Phys. 57 (1989) 53.

[11] J.A. Plascak, W. Figueiredo, B.C.S. Grandi, Braz. J. Phys. 29 (1999) 579.

[12] J. Chahine, J.R. Drugowich de Felício, N. Caticha, J. Phys. A 22 (1989) 1639.

[13] S. Wiseman, E. Domany, Phys. Rev. E 48 (1993) 4080.

[14] G. Kamieniarz, P. Kozlowski, R. Dekeyser, Phys. Rev. E 55 (1997) 3724.

[15] F.Y. Wu, K.Y. Lin, J. Phys. C 7 (1974) L181.

[16] E. Domany, E.K. Riedel, Phys. Rev. B 19 (1979) 5817.

[17] S. Bekhechi, A. Benyoussef, A. Elkenz, B. Ettaki, M. Loulid, Physica A 264 (1999) 503.

[18] M. Badehdah, S. Bekhechi, A. Benyoussef, M. Touzani, Physica B 291 (2000) 394.

[19] C.G. Bezerra, A.M. Mariz, J.M. de Araújo, F.A. da Costa, Physica A 292 (2001) 429.

[20] F.C. Alcaraz, C. Tsallis, J. Phys. A 15 (1982) 587.

[21] J.X. Le, Z.R. Yang, Phys. Rev. E 68 (2003) 066105.

[22] G.S. Grest, M. Widom, Phys. Rev. B 24 (1981) 6508.

[23] M.J. Martins, J.R.D. de Felicio, J. Phys. A 21 (1988) 1117.

[24] Y.Y. Goldschmidt, Phys. Rev. Lett. 56 (1986) 1627.

[25] J.R. Drugowich de Felicio, J. Chahine, N. Caticha, Phys. A 321 (2003) 529.

[26] G.N. Murthy, Phys. Rev. B 36 (1987) 7166.

[27] C. Tsallis, A.C.N. de Magalhães, Phys. Rep. 268 (1996) 306.

[28] A.N. Berker, S. Ostlund, J. Phys. C 12 (1975) 4961.

[29] M. Kaufman, R.B. Griffiths, Phys. Rev. B 24 (1981) 496. 